\title{
Symmetrized complex amplitudes for He double photoionization from the time-dependent close coupling and exterior complex scaling methods
}

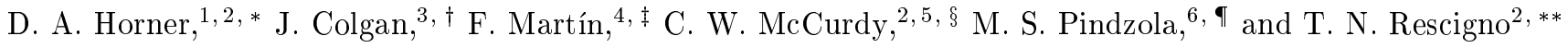 \\ ${ }^{1}$ Department of Chemistry, University of California, Berkeley, California 94720 \\ ${ }^{2}$ Lawrence Berkeley National Laboratory, Chemical Sciences, Berkeley, California 94720 \\ ${ }^{3}$ Theoretical Division, Los Alamos National Laboratory, Los Alamos, NM 87545, USA \\ ${ }^{4}$ Departamento de Química C-9, Universidad Autónoma de Madrid, 28049 Madrid, Spain. \\ ${ }^{5}$ Department of Applied Science, University of California, Davis, California 95616 \\ ${ }^{6}$ Department of Physics, Auburn University, Auburn, Alabama 36849, USA
}

(Dated: June 1, 2004)

\begin{abstract}
Symmetrized complex amplitudes for the double photoionization of helium are computed by the time-dependent close-coupling and exterior complex scaling methods, and it is demonstrated that both methods are capable of the direct calculation of these amplitudes. The results are found to be in excellent agreement with each other and in very good agreement with results of other $a b$ initio methods and experiment.
\end{abstract}

PACS numbers: $32.80 . \mathrm{Fb}, 34.10 .+\mathrm{x}$

In 1997 Malegat et al. [1, 2] derived a useful and compact representation of the triple differential cross section (TDCS) for double photoionization. That representation expresses the symmetry and kinematic aspects of the TDCS exactly, and explicitly displays the dynamical information in terms of a pair of complex amplitudes, $A_{g}\left(E_{1}, E_{2}, \theta_{12}\right)$ and $A_{u}\left(E_{1}, E_{2}, \theta_{12}\right)$,

$$
\begin{aligned}
\frac{d^{3} \sigma}{d E_{1} d \Omega_{1} d \Omega_{2}}= & \mid A_{g}\left(E_{1}, E_{2}, \theta_{12}\right)\left(\cos \theta_{1}+\cos \theta_{2}\right) \\
& +\left.A_{u}\left(E_{1}, E_{2}, \theta_{12}\right)\left(\cos \theta_{1}-\cos \theta_{2}\right)\right|^{2},
\end{aligned}
$$

where $E_{1}$ and $E_{2}$ are the energies of the exiting electrons, $\theta_{1}$ and $\theta_{2}$ are the angles they make with the polarization vector, and $\theta_{12}$ is the angle between their directions of ejection. The two amplitudes, $A_{g}$ and $A_{u}$, are called "symmetrized" because they are coefficients of the parts of the overall amplitude that are gerade and ungerade with respect to interchange of the electrons respectively. They depend on the energy sharing between the ejected electrons and the angle between them. For a given energy sharing, the dependence of these coefficients on the angle is generally simple and can be parameterized to a good approximation as a Gaussian function of $\theta_{12}$ [3]. The magnitudes of the two amplitudes and their relative phase can be extracted from fits to experiment, and make a convenient parameterization of the complete TDCS at a given energy sharing [4].

Because of the intrinsic spherical symmetry of the atomic problem, theoretical treatments of the double photoionization process for an atom are expressed -

\footnotetext{
*dahorner@lbl.gov

$\dagger$ jcolgan@lanl.gov

fernando.martin@uam.es

$\S$ cwmccurdy@lbl.gov

"pindula@physics.Aubur n.edu

**tnrescigno@lbl.gov
}

both formally and in computations - in terms of the coupled spherical harmonics, $y_{l_{1}, l_{2}}^{L, M}\left(\hat{k}_{1}, \hat{k}_{2}\right)$, (as functions of the directions of the final momenta, $\left.\left(\hat{k}_{1}, \hat{k}_{2}\right)\right)$, which are defined in terms of the ordinary spherical harmonics, $Y_{l, m}(\hat{k})$, by

$$
\begin{aligned}
& \sum_{m_{1}, m_{2}}^{L, M}\left(\hat{k}_{1}, \hat{k}_{2}\right)= \\
& \left.\sum_{1} m_{1} l_{2} m_{2} \mid l_{1} l_{2} L M\right) Y_{l_{1}, m_{1}}\left(\hat{k}_{1}\right) Y_{l_{2}, m_{2}}\left(\hat{k}_{2}\right)
\end{aligned}
$$

where $\left(l_{1} m_{1} l_{2} m_{2} \mid l_{1} l_{2} L M\right)$ is a vector coupling coefficient [5]. The key identity upon which Eq.(1) is based was derived originally by Kono and Hattori [6] and generalized by Malegat et al. $[1,2]$. For the case at hand of double photoionization of the helium ground state, for which the final state has $L=1$ and $M=0$, it is

$$
\begin{aligned}
y_{l, l+1}^{1,0}\left(\hat{k_{1}}, \hat{k_{2}}\right)=\frac{(-1)^{l}}{4 \pi} & \sqrt{\frac{3}{l+1}}\left(P_{l+1}^{\prime}\left(\cos \theta_{12}\right) \cos \theta_{2}\right. \\
& \left.-P_{l}^{\prime}\left(\cos \theta_{12}\right) \cos \theta_{1}\right) .
\end{aligned}
$$

where $P_{l}^{\prime}$ denotes the derivative of the Legendre Polynomial.

In recent calculations of helium double photoionization using the method of exterior complex scaling (ECS) with B-spline basis functions [7], we defined the triply differential cross section for double photoionization in terms of direct and exchange amplitudes. To relate those amplitudes to the symmetrized amplitudes in Eq.(1) is straightforward. Using the notation of reference [7], we can write the cross section in terms of coupled spherical harmonics as

$$
\begin{array}{r}
\frac{d^{3} \sigma}{d E_{1} d \Omega_{1} d \Omega_{2}}=\frac{4 \pi^{2}}{\omega c} \mid \sum_{l}\left(Q_{l l+1 k_{1} k_{2}}^{D} y_{l, l+1}^{1,0}\left(\hat{k_{1}}, \hat{k_{2}}\right)\right. \\
\left.+Q_{l l+1 k_{1} k_{2}}^{E} y_{l, l+1}^{1,0}\left(\hat{k_{2}}, \hat{k_{1}}\right)\right)\left.\right|^{2}
\end{array}
$$

with the direct and exchange amplitudes written in turn in terms of the amplitudes defined in Eqs.(30) and (31) 
of reference [7].

$$
\begin{gathered}
Q_{l_{1} l_{2} k_{1} k_{2}}^{D}=\sqrt{k_{1} k_{2}} i^{-\left(l_{1}+l_{2}\right)} e^{i \delta_{l_{1}}\left(k_{1}\right)+i \delta_{l_{2}}\left(k_{2}\right)} \mathcal{F}_{l_{1} l_{2} k_{1} k_{2}}^{d i r} \\
Q_{l_{1} l_{2} k_{1} k_{2}}^{E}=\sqrt{k_{1} k_{2}} i^{-\left(l_{1}+l_{2}\right)} e^{i \delta_{l_{1}}\left(k_{2}\right)+i \delta_{l_{2}}\left(k_{1}\right)} \mathcal{F}_{l_{1} l_{2} k_{1} k_{2}}^{e x c h} .
\end{gathered}
$$

where $\delta_{l}(k)$ is the Coulomb phase shift. By using Eq. (3) we can rearrange Eq.(4) to be of the form of Eq. (1). In this form, the symmetrized amplitudes are

$$
\begin{aligned}
A_{g / u}\left(E_{1}, E_{2}, \theta_{12}\right)=\frac{1}{2} & \sqrt{\frac{3}{\omega c}} \sum_{l} \frac{(-1)^{l}}{\sqrt{l+1}}\left[P_{l+1}^{\prime}\left(\cos \theta_{12}\right)\right. \\
& \left.\mp P_{l}^{\prime}\left(\cos \theta_{12}\right)\right] Q_{l l+1 k_{1} k_{2}}^{ \pm} .
\end{aligned}
$$

where the amplitudes $Q^{ \pm}$are defined as simple combinations of the direct and exchange ampludes,

$$
Q_{l_{1} l_{2} k_{1} k_{2}}^{ \pm}=\frac{1}{2}\left(Q_{l_{1} l_{2} k_{1} k_{2}}^{E} \pm Q_{l_{1} l_{2} k_{1} k_{2}}^{D}\right)
$$

Thus, with Eq.(6) we have expressed the symmetrized amplitudes, $A_{g / u}\left(E_{1}, E_{2}, \theta_{12}\right)$ in terms of the original direct and exchange amplitudes $\mathcal{F}_{l_{1} l_{2} k_{1} k_{2}}^{\text {dir } / \text { orch }}$ of the ECS study in reference [7].

We now turn to the time-dependent close-coupling (TDCC) theory describing double photoionization processes, which has been described in detail in previous work [8-10]. Following the method to calculate the TDCS described in reference [9], we can easily show how the TDCC approach is modified to calculate the gerade and ungerade amplitudes of Eq.(1).

We begin from Eq.(19) of reference [9] which expresses the TDCS in terms of the transformed two-dimensional momentum-space wavefunctions $P_{l_{1}, l_{2}}^{1}\left(k_{1}, k_{2}, t\right)$ for ${ }^{1} P$ symmetry:

$$
\begin{aligned}
\frac{d^{3} \sigma}{d \alpha d \Omega_{1} d \Omega_{2}}=\frac{\omega}{I} \frac{\partial}{\partial t} \frac{2}{\pi} \int_{0}^{\infty} d k_{1} \frac{2}{\pi} \int_{0}^{\infty} d k_{2} \delta & \left(\alpha-\tan ^{-1}\left(\frac{k_{2}}{k_{1}}\right)\right) \\
& \times\left|\sum_{l_{1}, l_{2}}(-i)^{l_{1}+l_{2}} e^{i\left(\delta_{l_{1}}\left(k_{1}\right)+\delta_{l_{2}}\left(k_{2}\right)\right)} P_{l_{1}, l_{2}}^{1}\left(k_{1}, k_{2}, t\right) y_{l_{1} l_{2}}^{1,0}\left(\hat{k_{1}}, \hat{k_{2}}\right)\right|^{2} .
\end{aligned}
$$

In this expression, $\alpha=\tan ^{-1}\left(\sqrt{E_{2} / E_{1}}\right)$ is the hyperspherical angle specifying the energy sharing, $\delta_{l}(k)$ is again the Coulomb phase shift, and integration over all solid angles and ejected energy gives, as required, the total integral cross section. We note that we integrate over all ejected energies $\left(0<E_{1}<E\right)$ to give the total integrated cross section.

Since the constraint on the sum over $l_{1}, l_{2}$ is that they must couple to give $L=1$ (for final ${ }^{1} P$ symmetry) we can replace the sum in eq. (8) with a single sum over $l$ where we now write $l_{1}, l_{2}$ as $l, l+1$. We also see that symmetry arguments allow us to write

$$
\begin{aligned}
y_{l, l+1}^{1,0}\left(\hat{k_{1}}, \hat{k_{2}}\right) & =y_{l+1, l}^{1,0}\left(\hat{k_{2}}, \hat{k_{1}}\right) \\
P_{l, l+1}^{1} P & \left(k_{1}, k_{2}, t\right)=P_{l+1, l}^{1 P}\left(k_{2}, k_{1}, t\right),
\end{aligned}
$$

so that the expression inside the square of Eq. (8) may be expressed as

$$
\begin{aligned}
& \sum_{l}(-i)^{2 l+1} \frac{(-1)^{l}}{4 \pi} \sqrt{\frac{3}{l+1}} \times \\
& \left\{\operatorname { c o s } \theta _ { 2 } \left[e^{i\left(\delta_{l}\left(k_{1}\right)+\delta_{l+1}\left(k_{2}\right)\right)} P_{l, l+1}^{1} P\left(k_{1}, k_{2}, t\right) P_{l+1}^{\prime}\left(\cos \theta_{12}\right)\right.\right. \\
& \left.-e^{i\left(\delta_{l+1}\left(k_{1}\right)+\delta_{l}\left(k_{2}\right)\right)} P_{l, l+1}^{1} P\left(k_{2}, k_{1}, t\right) P_{l}^{\prime}\left(\cos \theta_{12}\right)\right] \\
& +\cos \theta_{1}\left[e^{i\left(\delta_{l+1}\left(k_{1}\right)+\delta_{l}\left(k_{2}\right)\right)} P_{l, l+1}^{1} P\left(k_{2}, k_{1}, t\right) P_{l+1}^{\prime}\left(\cos \theta_{12}\right)\right. \\
& \left.\left.-e^{i\left(\delta_{l}\left(k_{1}\right)+\delta_{l+1}\left(k_{2}\right)\right)} P_{l, l+1}^{1} P\left(k_{1}, k_{2}, t\right) P_{l}^{\prime}\left(\cos \theta_{12}\right)\right]\right\}
\end{aligned}
$$

After some manipulation, and by writing

$$
\begin{aligned}
& P_{l, l+1}^{ \pm}\left(k_{1}, k_{2}, t\right)=\frac{1}{2}\left[e^{i\left(\delta_{l}\left(k_{1}\right)+\delta_{l+1}\left(k_{2}\right)\right)} P_{l, l+1}^{1} P\left(k_{1}, k_{2}, t\right)\right. \\
& \left. \pm e^{i\left(\delta_{l+1}\left(k_{1}\right)+\delta_{l}\left(k_{2}\right)\right)} P_{l, l+1}^{1} P\left(k_{2}, k_{1}, t\right)\right]
\end{aligned}
$$

we can finally reduce the expression inside the square in Eq.(8) to the form

$$
\begin{aligned}
a_{g}\left(E_{1}, E_{2}, \theta_{12}\right) & \left(\cos \theta_{1}+\cos \theta_{2}\right) \\
& +a_{u}\left(E_{1}, E_{2}, \theta_{12}\right)\left(\cos \theta_{1}-\cos \theta_{2}\right)
\end{aligned}
$$



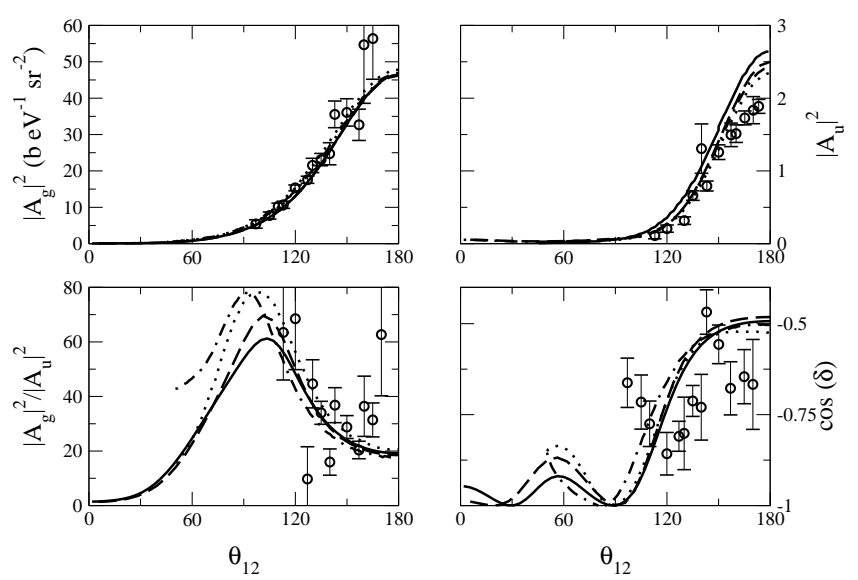

FIG. 1: Amplitudes for photon energy $40 \mathrm{eV}$ above threshold with $E_{1}=5 \mathrm{eV}$. Solid curve: present result, ECS. Dashed curve: Present result, TDCC. Dotted curve: CCC [4]. Dashdotted curve: HRM-SOW [4]. Circles: experiment of reference [4]

where

$$
\begin{aligned}
& a_{g / u}\left(E_{1}, E_{2}, \theta_{12}\right)= \\
& \quad \pm \sum_{l}(-i)^{2 l+1} \frac{(-1)^{l}}{4 \pi} \sqrt{\frac{3}{l+1}} \\
& \quad\left(P_{l+1}^{\prime}\left(\cos \theta_{12}\right) \mp P_{l}^{\prime}\left(\cos \theta_{12}\right)\right) P_{l, l+1}^{ \pm}\left(k_{1}, k_{2}, t\right) .
\end{aligned}
$$

Finally, we can relate the amplitudes $A_{g / u}$ of Eq.(1) to the amplitudes $a_{g / u}$ in Eq.(13)

$$
\begin{aligned}
A_{g / u}= & \frac{\omega}{I} \frac{\partial}{\partial t} \frac{2}{\pi} \int_{0}^{\infty} d k_{1} \frac{2}{\pi} \int_{0}^{\infty} d k_{2} \\
& \delta\left(\alpha-\tan ^{-1}\left(\frac{k_{2}}{k_{1}}\right)\right) a_{g / u}\left(E_{1}, E_{2}, \theta_{12}\right) .
\end{aligned}
$$

These are the symmetrized amplitudes from the TDCC approach that we can compare with those calculated by the ECS and other methods.

In the calculations using the TDCC and ECS methods we report here, we have used the velocity gauge throughout, although the results in length and velocity gauges are in excellent agreement for both theories. In Fig. 1 we show symmetrized amplitudes for double photoionization of helium at $40 \mathrm{eV}$ above threshold for the energy sharing arrangement, $E_{1}=5 \mathrm{eV}$ and $E_{1}=35 \mathrm{eV}$. Along with the current TDCC and ECS results, we show the results of previously reported calculations using the convergent close coupling (CCC) method and hyperspherical $R$-matrix method with semi-classical outgoing waves (HRM-SOW) [4]. The agreement is very good overall, although one notes some differences in both ratio of amplitudes and their relative phases when either $A_{g}$ or $A_{u}$, or both, are very small. Note that the $\mathrm{CCC}$ and $\mathrm{H} R \mathrm{M}-$ SOW results were only reported for angles larger than $\theta_{12}=45^{\circ}$. Experimental results from reference [4] are also shown in Fig. 1, and it is clear that the experiment
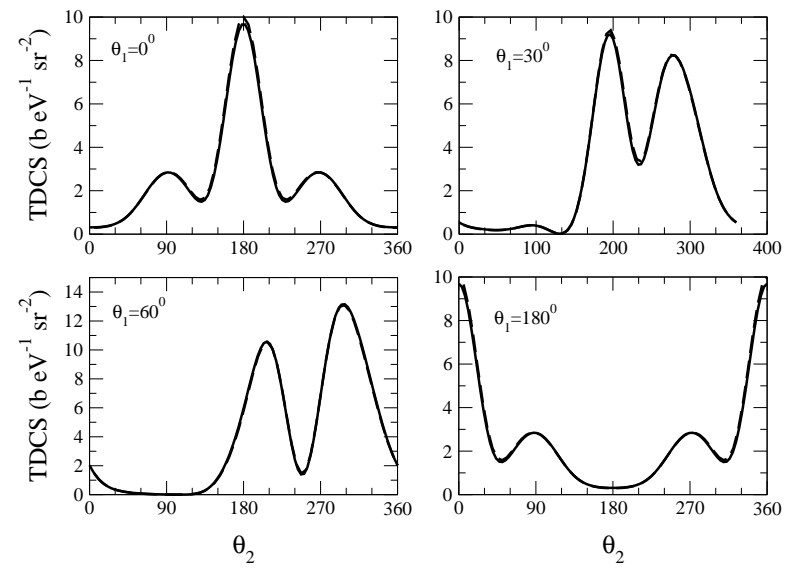

FIG. 2: TDCS for photon energy $40 \mathrm{eV}$ above threshold with $E_{1}=5 \mathrm{eV}$ for various ejection angles, $\theta_{1}$. Solid curve: present result, ECS. Dashed curve:Present result, TDCC.

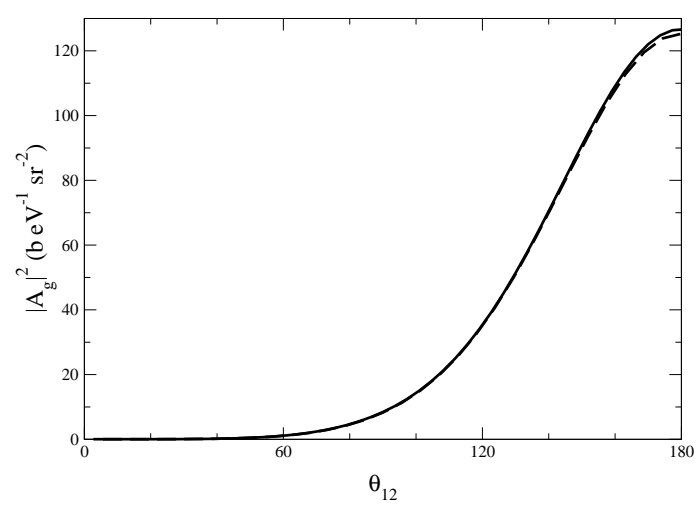

FIG. 3: Amplitude for photon energy $20 \mathrm{eV}$ above threshold with $E_{1}=E_{2}=10 \mathrm{eV}$, equal energy sharing. Solid curve: present result, ECS. Dashed curve:Present result, TDCC.

is not able to distinguish between the theories. Note that not all the experimental points fit on the expanded scales used to show the differences in the theoretical calculations in Fig. 1.

With these computed amplitudes, Eq. (1) gives us an expression for the TDCS, so it is natural to ask what the consequences might be of the small differences seen in the symmetrized amplitudes in Fig. 1 when they are used to compute the TDCS. The TDCS is shown in Fig. 2 for the energy sharing of Fig. 1 as a function of $\theta_{2}$ for various values of $\theta_{1}$. In spite of the differences that can be seen in the amplitudes, the cross sections computed using the TDCC and ECS methods are in essentially perfect agreement. We also note that TDCS values calculated using TDCC [10] and ECS [7] have been previously published, and that here the TDCC results have been multiplied by 2 in order to compare with the ECS calculations, due to the different conventions in the definition of the single differential cross section.

The case of equal energy sharing between the two ejected electrons is unique, in that the ungerade ampli- 

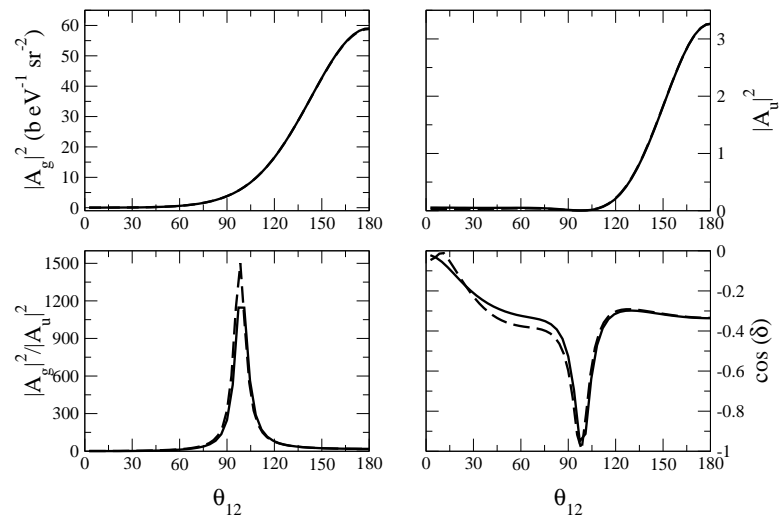

FIG. 4: Amplitudes for photon energy $20 \mathrm{eV}$ above threshold, with $E_{1}=19 \mathrm{eV}$. Solid curve: present result, ECS. Dashed curve:Present result, TDCC.
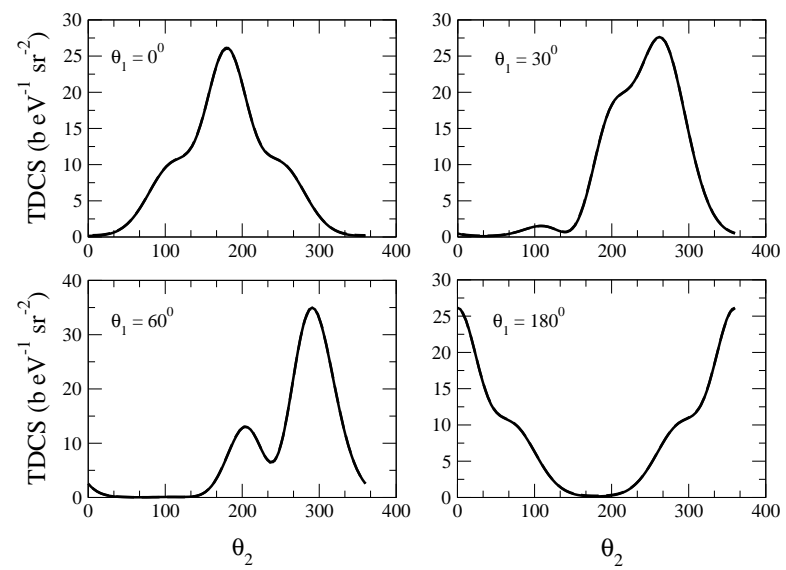

FIG. 5: TDCS for photon energy $20 \mathrm{eV}$ above threshold, with $E_{1}=19 \mathrm{eV}$ for various ejection angles $\theta_{1}$. Solid curve: present result, ECS. Dashed curve (essentially identical to solid curve):Present result, TDCC.

tude, $A_{u}$, is identically zero for all relative angles $\theta_{12}$. In Fig. 3 we show the gerade amplitude, $A_{g}$ for equal en- ergy sharing with photon energy $20 \mathrm{eV}$ above threshold. Again there is essentially perfect agreement between the ECS and TDCC results.

It is particularly interesting to ask what the symmetrized amplitudes look like for extremely unequal energy sharing. We show the amplitudes for the case of $E_{1}=19 \mathrm{eV}$ and $E_{2}=1 \mathrm{eV}$ in Fig. 4 at a photon energy $20 \mathrm{eV}$ above the double ionization threshold. The relative phase shows a sharp feature as a function of $\theta_{12}$ as does the ratio of the magnitudes of the amplitudes, and there are some small, but visible differences between the TDCC and ECS results. However, when one examines the corresponding set of TDCS curves in Fig. 5 one sees that, once again, the ECS and TDCC methods agree with one another essentially exactly.

This study has shown that both the TDCC method and the ECS method can be used to compute the complex symmetrized amplitudes for the double photoionization process that provide a convenient and compact way to parameterize the TDCS. The results of calculations using these two methods are generally in better agreement with each other than they are with the results of CCC or HRM-SOW calculations, although all four methods describe the physics of this problem well.

\section{Acknowledgments}

Work performed at the University of California Lawrence Berkeley National Laboratory was under the auspices of the US Department of Energy under Contract DE-AC03-76SF00098 and was supported by the U.S. DOE Office of Basic Energy Sciences, Division of Chemical Sciences. Work at LANL was performed under the auspices of the U.S. Department of Energy through the Theoretical Division at the Los Alamos National Laboratory. DH is supported by a US DOE Computational Science Graduate Fellowship. FM is supported by the DGI (Spain) project No. BFM2003-00194.
[1] L. Malegat, P. Selles, and A. Huetz, J. Phys. B: At. Mol. Opt. Phys. 30, 251 (1997).

[2] L. Malegat, P. Selles, P. Lablanquie, J. Mazeau, and A. Huetz, J. Phys. B: At. Mol. Opt. Phys. 30, 263 (1997).

[3] A. S. Kheifets and I. Bray, Phys. Rev. A 65, 022708 (2002).

[4] P. Bolognesi, A. S. Kheifets, I. Bray, L. Malegat, P. Selles, A. K. Kazansky, and L. Avaldi, J. Phys. B: At. Mol. Opt. Phys. 36, L241 (2003).

[5] A. R. Edmonds, Angular Momentum in Quantum Mechanics (Princeton University Press, Princeton, 1957), p. $37 \mathrm{ff}$.

[6] A. Kono and S. Hattori, Phys. Rev. A 29, 2981 (1984).

[7] C. W. McCurdy, D. A. Horner, T. N. Rescigno, and F. Martín, Phys. Rev. A 69, 032707 (2004).

[8] M. S. Pindzola and F. Robicheaux, Phys. Rev. A 57, 318 (1998).

[9] J.Colgan, M. S. Pindzola, and F. Robicheaux, J. Phys. B: At. Mol. Opt. Phys. 34, L457 (2001).

[10] J. Colgan and M. S. Pindzola, Phys. Rev. A 65, 032729 (2002). 